\title{
Higher fatty liver index is associated with increased risk of new onset heart failure in healthy adults: A nationwide population-based study in Korea
}

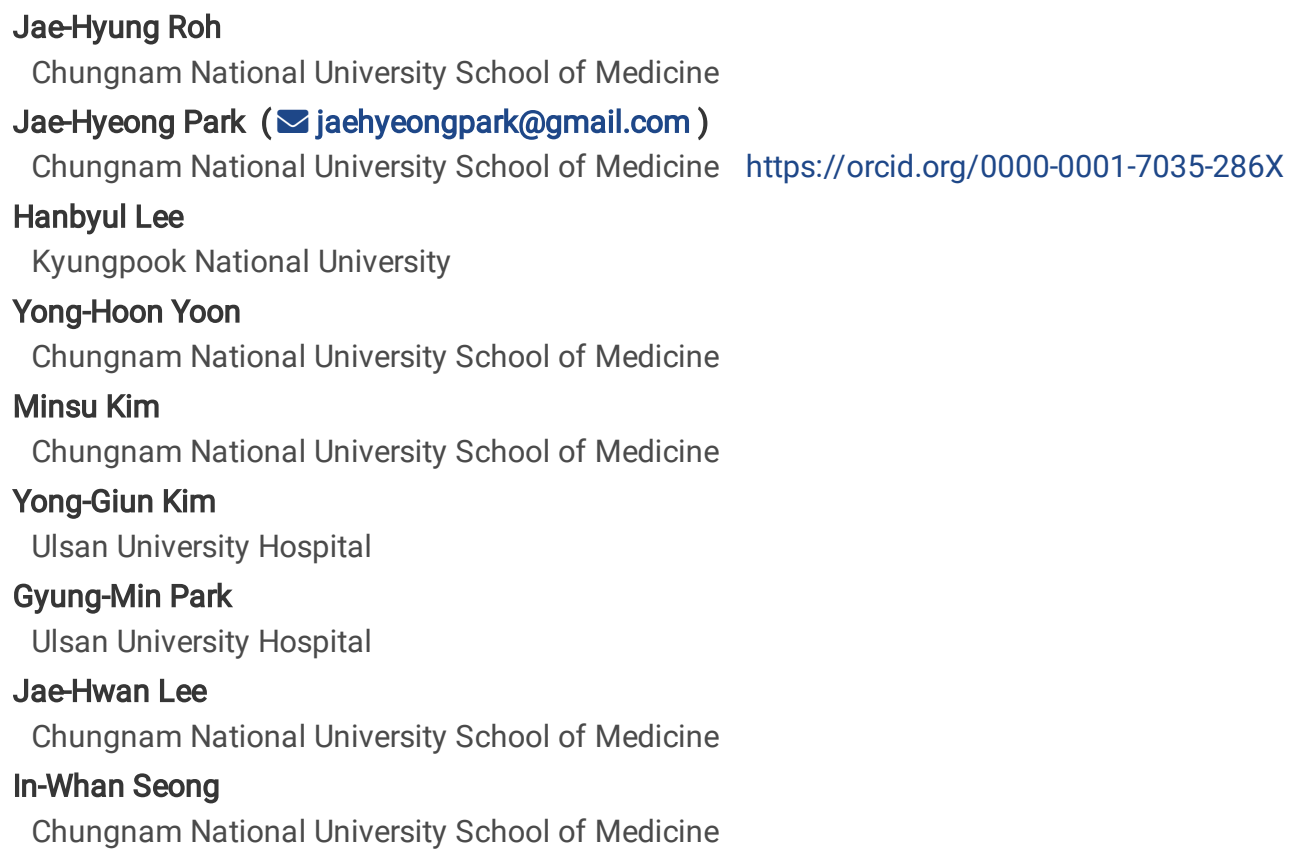

\section{Research article}

Keywords: Fatty Liver Index, Non-alcoholic Fatty Liver Disease, Heart failure, Healthy people programs

Posted Date: February 13th, 2020

DOl: https://doi.org/10.21203/rs.2.17577/v3

License: (c) (i) This work is licensed under a Creative Commons Attribution 4.0 International License. Read Full License

Version of Record: A version of this preprint was published at BMC Cardiovascular Disorders on April 28th, 2020. See the published version at https://doi.org/10.1186/s12872-020-01444-x. 


\section{Abstract}

Background Heart failure (HF) is relatively common cardiovascular disease with high mortality and morbidity. Although it is associated with many cardiovascular risk factors, the association between nonalcoholic fatty liver disease (NAFLD), the most common chronic liver disease, and HF has not been evaluated in a large-scale cohort study. Thus, we evaluated the ability of the fatty liver Index (FLI), a surrogate marker of NAFLD, to predict the development of HF in healthy individuals.

Methods We evaluated 308,578 healthy persons without comorbidities who underwent the National Health check-ups in the republic of Korea from 2009 to 2014. The association between the FLI and HF was analyzed using multivariate Cox proportional-hazards models.

Results During a median of 5.4 years' follow-up, 2,532 subjects $(0.8 \%)$ were newly diagnosed with HF. Subjects were categorized into quartile groups according to FLI (Q1, 0-4.9; Q2, 5.0-12.5; Q3, 12.6-31.0; and Q4, >31.0). The cumulative incidence of HF was significantly higher in subjects with the highest FLI than in those with the lowest FLI (Q1, 307 [0.4\%] and Q4, 890 [1.2\%]; P<0.001). Adjusted hazard ratios (HRs) indicated that the highest FLI was independently associated with an increased risk for HF (HR between Q4 and Q1, 2.709; 95\% confidence interval=2.380-3.085; $\mathrm{P}<0.001)$. FLI was significantly associated with increased risk of new-onset HF regardless of their baseline characteristics.

Conclusions Higher FLI was independently associated with increased risk of HF in healthy Korean population.

\section{Introduction}

Non-alcoholic fatty liver disease (NAFLD) is the most common form of chronic liver disease. Its estimated prevalence is $15-30 \%$ in general population [1]. The prevalence of NAFLD has been increasing along with an increased incidence of obesity, insulin resistance, and non-insulin dependent diabetes, and metabolic syndrome [2]. NAFLD can be found in 60-70\% among obese or diabetic patients [1]. Many previous studies reported a positive association between NAFLD and established cardiovascular risk factors, including abdominal obesity, dyslipidemia, insulin resistance, and hypertension [3]. These cardiovascular risk factors are also components of metabolic syndrome. NAFLD is regarded as the hepatic manifestation of metabolic syndrome. In patients with NAFLD, cardiovascular disease is the third most common cause of death after extrahepatic malignancies and liver-related complications $[4,5]$.

Heart failure (HF) is one of the major cardiovascular diseases with high morbidity, mortality, which exerts a high cost on healthcare systems globally $[6,7]$. The prevalence of $\mathrm{HF}$ is approximately $1-2 \%$ in the adult population in developed countries, and its increasing prevalence is more than $10 \%$ among people over 70 years of age $[6,8]$. HF and NAFLD often coexist due to their common risk factors and similar pathophysiological processes $[9,10]$. HF alone may cause liver disease and vice versa in the absence of other cardiovascular risk factors [10].

Several previous studies with a small sample size on co-existing $\operatorname{NAFLD}$ and HF $[11,12]$ reported a simple association in cross-sectional analysis. In this study, a large cohort of healthy Korean adults without known cardiovascular risk factors and co-morbidities was analyzed to evaluate the association between NAFLD and new-onset HF.

\section{Methods}

\section{Data sources}

The National Health Insurance Service-National Sample Cohort 2.0 (NHIS-NSC 2.0) data set, which comprises more than 97\% of Korean citizens affiliated with the obligatory public health insurance program of the National Insurance Health Service (NIHS) was analyzed for this study. The total population ( $n=48,222,537)$ was classified into 2,142 groups according to their age, sex, residing cities, eligible status, and income levels. From each stratum, randomly selected $2.1 \%$ individuals $(n=1,021,208)$ in the year of 2006 were included in NHIS-NSC 2.0 data. In addition, retrospective and prospective follow-up data collected from 2002 to 2015 were included in the data set [13]. The individuals included in NHIS-NSC 2.0 are representative of the entire Korean population.

The cohort data was composed of four data sets: (1) sociodemographics; (2) medical claims including information on diagnosis based on the 10th revision of the International Classification of Disease (ICD-10) codes, admission, and treatment; (3) the results of the National Health Screening; (4) information on the medical institutions. The Korean national health check-ups comprise of the questionnaires on medical history and health-related behaviors such as smoking and alcohol consumption, chest X-rays, physical examinations, and blood tests. About $72.1 \%$ of the eligible population had completed the National Health screening programs, according to the $2013 \mathrm{NHIS}$ statistics [13]. The cohort data also included mortality statistics from the death registration database of Statistics Korea. 
The study protocol was approved by the NHIS review committee for accessing NHIS-NSC 2.0 data and also approved by the Institutional Review Board of the Chungnam National University Hospital in Daejeon, Korea (IRB No. 2019-10-053). The board waivered the requirement for informed consent, for NHIS-NSC 2.0 data was public.

\section{Study population}

Study subjects were any Korean citizens who had completed the National Health check-up at least once from 2009 to 2014 . Data from the first check-up was defined as the index data, and the year when the index check-up was done was the index year. Subjects with age ${ }^{3} 20$ years old were included, whereas subjects meeting the pre-specified exclusion criteria were excluded. All subjects previously diagnosed with HF were excluded for the purpose of the study. Other exclusion criteria were comorbid conditions that could affect the onset of HF, including hypertension, diabetes, atrial fibrillation, cerebrovascular disease, ischemic heart disease, peripheral vascular disease, valvular heart disease, chronic kidney disease, and chronic pulmonary disease. Subjects on prescribed medications such as oral hypoglycemic agents, antihypertensives, or lipid-lowering drugs within one year before the index check-up, subjects with hypertension (systolic blood pressure ${ }^{3}$ $140 \mathrm{mmHg}$ or diastolic blood pressure ${ }^{3} 90 \mathrm{mmHg}$ ), and subjects with elevated fasting blood glucose ${ }^{3} 126 \mathrm{mg} / \mathrm{dL}$ at the index check-up were excluded. Subjects with risk factors that could affect the FLI, such as liver disease and autoimmune disease diagnosed before the index year, were excluded. Finally, those with any missing data in the index check-up were also excluded.

\section{Definition of HF}

The primary outcome of this study was HF incidence in relation to the FLI. The incidence of HF was defined as the first HF occurrence documented on the medical record at least two separate days of outpatient visits and admission, or one-time record of death due to heart failure. The diagnosis of HF was screened in data from questionnaires and the 1-year medical claim data before the index year. The presence of HF was defined using the following ICD-10 codes in the medical claim dataset: "Hypertensive heart disease with HF" (I10.0), "Hypertensive heart disease with hypertensive kidney disease with HF" (I13.0), "Hypertensive heart disease with hypertensive kidney disease with HF and kidney failure" (113.2), "Ischemic cardiomyopathy” (I25.5), "Dilated cardiomyopathy” (142.0), "Cardiomyopathy, unspecified” (I42.9), "Cardiomyopathy in diseases classified elsewhere" (143), "HF" (I50) including "Congestive HF" (I50.0), "Left ventricular failure" (I50.1), and "HF, unspecified" (I50.9).

\section{Definition/Ascertainment of covariates}

Body mass index (BMI) was weight $(\mathrm{kg})$ over height $(\mathrm{m})$-squared. The population with a BMI of $\geq 25 \mathrm{~kg} / \mathrm{m}^{2}$ was regarded as obese according to the World Health Organization guideline for the Asian population [14]. Smoking status was classified into three categories: nonsmoker, ex-smoker, and current smoker. Alcohol consumption and physical activity were reported using standard self-reporting questionnaires. The total amount of alcohol consumption was calculated by multiplying the number of days per week of drinking and the amount of alcohol consumed per day. Levels of physical activity were estimated by summing up the intensity levels of workout in METs multiplied by the number of days per week of each intensity level : (1) 30-min of light exercise (2 METs); (2) 30-min of moderate exercise (3 METs); (3) 20-min of vigorous exercise (6 METs).

Blood samples were analyzed in a number of qualified institutions, which were annually audited by the Korean Association of External Quality Assessment Service. Data were reviewed at the time of HF occurrence, disqualification of the NHIS (death or immigration), or the end of the study (December 31th, 2015).

\section{Calculation of the fatty liver index}

The FLI is a well-validated surrogate marker for identifying patients with NAFLD [13]. The FLI was calculated with triglycerides (TG), BMI, gamma-glutamyl transferase (GGT), and waist circumference (WC) with the following equation:

$F L I=\left(e^{0.953^{\prime} \log _{e}(T G)+0.139^{\prime} B M I+0.718^{\prime} \log _{e}(G G T)+0.053^{\prime} W C-15.745}\right) /\left(1+e^{0.953^{\prime} \log _{e}(T G)+0.139^{\prime} B M I+0.718^{\prime} \log _{e}(G G T)+0.053^{\prime} W C-15.745}\right)^{\prime} 100$

The original study on the FLI used 60 as the cutoff value, and the FLIs higher than 60 indicated fatty liver with a positive likelihood ratio of 4.3 in the general population [15]. Although the FLI is simple to calculate and easy to screen fatty liver disease, there has been insufficient 
evidence to use the same value in Asians because of lower BMI and WC than other ethnic populations [16]. In this study, instead of using the absolute value of the calculated FLI, subjects were grouped into quartiles according to their FLI range, and each quartile group was used in the statistical analysis to determine the relationship between FLI and new-onset HF.

\section{Statistical analysis}

Continuous variables were used as mean \pm standard deviation and categorical parameters as the numbers with a percentage. All statistical analyses were performed using R software version 3.3.3 (R Foundation for Statistical Computing, Vienna, Austria; www.r-project.org). Statistical differences between the FLI quartiles were estimated using the Chi-square test and one-way analysis of variance tests. Cumulative event rates of the FLI quartiles were calculated with the Kaplan-Meier method and compared with each other using a log-rank test. Adjusted hazard ratios (HR) and 95\% confidence interval (Cl) for HF incidence were estimated using Cox proportional hazard regression analysis. In the multivariate analysis, age and sex were adjusted in Model 1. In Model 2, clinical characteristics associated with new-onset HF of borderline statistical significance $(\mathrm{P}<0.100)$ were adjusted in addition to age and sex. Confounding factors, such as hypertension and diabetes, were excluded because of their strong association with NAFLD. Using NAFLD with other risk factors in a multivariate model raises the possibility of introducing multicollinearity into the model. The variance inflation factor (VIF) in all models (Supplementary Table 1) was examined, and VIF levels were less than 10 , which resolved the multicollinearity. P-values of $<0.05$ were considered statistically significant.

\section{Results}

\section{Baseline characteristics of the participants}

A total of 308,578 subjects were analyzed after excluding 248,306 subjects who met the exclusion criteria. The number of subjects matching each exclusion criteria was presented in Figure 1. Study population was divided into four groups according to their FLI quartile values; first quartile (Q1) of the FLI = 0 - 4.9; second quartile (Q2) of the FLI = 5.0 - 12.5; third quartile (Q3) of the FLI = 12.6 - 31.0; and fourth quartile (Q4) of the FLI >31.0. The comparisons of baseline clinical characteristics and laboratory findings according to the FLI quartiles were summarized in Table 1. The subjects with higher FLIs had a trend toward older ages and a higher proportion of males.

Numbers of subjects with high BMI, high waist circumference, hypertension, and a heavier amount of alcohol consumption, and a proportion of current smokers tended to increase from Q1 to Q4. Serum levels of fasting glucose, total cholesterol, triglyceride, and LDL cholesterol were increasing from Q1 to Q4.

\section{Association between FLI and the incidence of HF}

The median follow-up duration was 5.4 years (interquartile range: 4.1 - 6.3 years), and 2,532 subjects (0.8\%) had new-onset HF. Table 2 shows the result of the univariate analysis of new-onset HF. Male gender, older age, higher BMI, higher total cholesterol, higher TG, and lower HDL cholesterol levels were significantly associated with new-onset HF. Cumulative incidences of new-onset HF, by the FLI quartiles, were presented in Figure 2. The incidence of new-onset HF was significantly higher in subjects with higher FLIs, compared to those with lower FLIs (Q1, 307 [0.4\%]; Q2, 543 [0.7\%]; Q3, 792 [1.0\%]; and Q4, 890 [1.2\%], P<0.001 by the Log-rank test). In the multivariate models, the model 1 was adjusted for age, and the model 2 was for clinical characteristics with borderline statistical significance in addition to age and sex; the association between the FLI quartile and HF incidence remained statistically significant in each model in the time-dependent Cox proportional hazard analysis (Table 3). Drawn from the previous studies, specific ranges of the FLI cutoff values were used to categorize the study population ( $0 \leq$ $\mathrm{FLI}<30,30 \leq \mathrm{FLI}<60$, and FLI $\geq 60$, by Bedogni et al. [13]; $0 \leq \mathrm{FLI}<25,25 \leq \mathrm{FLI}<35$, and FLI $\geq 35 \mathrm{for}$ male, $0 \leq \mathrm{FLI}<10,10 \leq \mathrm{FLI}<20$, and $\mathrm{FLI} \geq 20$ for female, by Yang et al. [15]). Regardless of the FLI ranges, a group with the highest FLI had the highest risk for new-onset HF (Supplementary Table 1).

\section{Subgroup analysis}

The adjusted HRs of subgroups were summarized in Figure 3. The FLI was a significant determinant of new-onset HF in all subgroups evaluated(Figure 3).

\section{Discussion}


This study showed that a higher FLI was associated with an increased risk of new-onset HF in the healthy Korean adult population. Multivariate models were adopted to control several confounding factors such as hypertension and diabetes, which are well-known risk factors for HF. A more direct association between NAFLD and HF was evaluated by excluding hypertension and diabetes form the multivariate models, and the association was found in most subgroups.

The association between fatty liver and HF has been observed in several studies [11, 12]. Mildly elevated serum GGT level in the absence of excessive alcohol consumption was found to be a long-term, independent predictor of incident HF in large population-based observational studies [18-20]. Zhang et al. reported that 37 patients had NAFLD among 102 patients with HF with reduced ejection fraction (HFrEF) (36.3\%) screened with ultrasonography [12]. In the Framingham study, a high borderline level of GGT, even within the normal range, was associated with an increased risk of HF by 1.71 times (95\% $\mathrm{Cl}=1.21-2.41)$, compared to less than median levels of GGT [18]. The use of GGT levels improved risk re-classification modestly (net reclassification index, 5.7\%; $\mathrm{P}=0.01$ ) in the HF prediction [18]. In a prospective cohort study in Finland, including 18,353 men and 19,726 women of 25-74 years of age, moderate to high levels of serum GGT (from the 50th to the 90 th percentiles) were significantly associated with the incidence of HF. The predictive power of serum GGT in association with HF was stronger in participants aged less than 60 years [20].

The presence of NAFLD was associated with higher BMI, higher left ventricular (LV) mass index, and more severe LV fibrosis [12]. Other studies showed that patients with NAFLD had increased LV wall thickness and varying degrees of subclinical LV systolic or diastolic dysfunction [21, 22]. Increased LV wall thickness and fibrosis are markers of LV diastolic dysfunction regardless of symptoms. Jung et al. showed that groups with mild and moderate to severe NAFLD had a higher risk of abnormal LV relaxation compared to normal controls [21]. In cohort study of 20,821 healthy Korean adults, odds ratios for abnormal LV relaxation (mild group: 1.29 [95\% Cl of 1.15 - 1.46]; moderate to severe group: 1.95 [95\% Cl of $1.61-2.35]$ ) and odds ratios for relative wall thickness greater than $0.42 \mathrm{~cm}$ (mild group: 1.26 [95\% $\mathrm{Cl}$ of 1.05 1.52]; moderate to severe group: 1.46 [95\% $\mathrm{Cl}$ of $1.08-1.95])$ both increased.

The proposed mechanisms of increased LV wall thickness include insulin resistance [23], increased activity of the renin-angiotensinaldosterone system (RAAS) [24], and endothelial dysfunction and inflammation [11]. Insulin resistance may contribute to the development of LV hypertrophy and HF through increased renal sodium retention and activation of the sympathetic nervous system [25, 26]. Increased activity of the sympathetic nervous system can also accelerate the process of hepatic fibrosis [27]. Mediators of the RAAS, especially angiotensin II and aldosterone, play important roles in the development of hypertension and HF. Angiotensin II can also be expressed by activated human hepatic stellate cells [24]. Activated RAAS causes hepatic injury and induces fibrosis through angiotensin II-mediated stimulation of fibroblast proliferation and release of inflammatory cytokines [28]. Endothelial dysfunction, in part of the inflammatory process, can increase arterial stiffness and LV afterload through increased vascular tone, sympathetic overactivity, and sodium retention [29]. Inflammation can promote coronary atherosclerosis and increase the risk for cardiomyopathy and conduction abnormalities [11]. Inflammatory mediators also affect the progression of fatty liver disease via impaired fatty acid oxidation, increased oxidative stress, and local inflammation [30].

NAFLD was associated with poor prognosis in chronic and acute HF patients [31, 32]. Although the exact mechanism of this remains unclear, there are several proposed explanations; increased coronary atherosclerosis, LV hypertrophy and dysfunction, and aortic valve calcification [33-35]. Because the liver plays an important role in regulating antioxidant and anti-inflammatory processes [36], hepatic dysfunction can aggravate chronic inflammation and oxidative tissue injury in HF patients [37]. These impaired immunological processes through the hepatic injury may further deteriorate the prognosis of HF patients [38].

\section{Limitations}

This study has several limitations. First, the accuracy of HF diagnosis was not validated in this study. The records of medical claims and the National Health Screening data were used for identifying HF patients, and the incidence of new-onset HF was estimated only using the NHISNSC 2.0 data based on the ICD-10 code. If the information on echocardiographic imaging and serum biomarkers, including B-type natriuretic peptide or N-terminal pro-B-type natriuretic peptide, were available, the diagnosis of HF would have been more accurate. Further studies are needed to identify HF patients more accurately when using national health insurance data. Second, this study is observational and could not identify other contributable factors in the development of HF in relation to fatty liver. Third, $45 \%$ of the participants in the cohort were excluded due to their comorbidities, and this could affect the representativeness and generalizability of the findings. Finally, this study included only Koreans, and caution should be taken in extrapolating the study's conclusion to other ethnic populations.

\section{Conclusions}

In the healthy Korean population, higher FLI was independently associated with increased risk for new-onset HF. 


\section{Clinical perspectives}

Identification of increased HF risk may have important public health implications because the management of metabolic syndrome and prevention of HF are important in reducing cardiovascular morbidity and mortality. Further studies are needed to document the effect of management of NAFLD, not only on the risk of new-onset HF but also on the risk reduction of cardiovascular morbidity and mortality.

\section{Declarations}

\section{Ethics approval and consent to participate}

Institutional Review Board of Chungnam National University Hosptial approved this study protocol (IRB No. 2019-10-053). Our IRB waived the requirement for informed consent.

\section{Consent for publication}

Not applicable

\section{Availability of data and material}

The datasets used and analyzed during the present study are available from the National Health Insurance Service. It can be used after the acquisition of the right to use this data.

\section{Competing interests}

None. The authors have nothing to declare.

\section{Funding}

This work was supported by the research fund of Chungnam National University

\section{AUTHOR CONTRIBUTIONS}

RJH and PJH: study conception and design, acquisition of data, interpretation of data, drafting of the manuscript, and critical revision; LHB: acquisition of data, analysis and interpretation of data; and YYH, KMS, KYG, PGM, LJH, and SIW: interpretation of data, and critical revision.

\section{References}

1. Targher G, Day CP, Bonora E. Risk of cardiovascular disease in patients with nonalcoholic fatty liver disease. N Engl J Med. 2010;363:1341-50.

2. McPherson S, Hardy T, Henderson E, Burt AD, Day CP, Anstee QM. Evidence of NAFLD progression from steatosis to fibrosingsteatohepatitis using paired biopsies: implications for prognosis and clinical management. J Hepatol. 2015;62:1148-55.

3. Angulo P. Nonalcoholic fatty liver disease. N Engl J Med. 2002;346:1221-31.

4. Ekstedt M, Hagstrom $H$, Nasr P, Fredrikson M, Stal P, Kechagias S, et al. Fibrosis stage is the strongest predictor for disease-specific mortality in NAFLD after up to 33 years of follow-up. Hepatology. 2015;61:1547-54.

5. Angulo P, Kleiner DE, Dam-Larsen S, Adams LA, Bjornsson ES, Charatcharoenwitthaya P, et al. Liver Fibrosis, but No Other Histologic Features, Is Associated With Long-term Outcomes of Patients With Nonalcoholic Fatty Liver Disease. Gastroenterology. 2015;149:389-97 e10.

6. Ponikowski P, Voors AA, Anker SD, Bueno H, Cleland JGF, Coats AJS, et al. 2016 ESC Guidelines for the diagnosis and treatment of acute and chronic heart failure: The Task Force for the diagnosis and treatment of acute and chronic heart failure of the European Society of 
Cardiology (ESC)Developed with the special contribution of the Heart Failure Association (HFA) of the ESC. Eur Heart J. 2016;37:21292200.

7. Lee JH, Kim MS, Kim EJ, Park DG, Cho HJ, Yoo BS, et al. KSHF Guidelines for the Management of Acute Heart Failure: Part I. Definition, Epidemiology and Diagnosis of Acute Heart Failure. Korean Circ J. 2019;49:1-21.

8. Lee JH, Lim NK, Cho MC, Park HY. Epidemiology of Heart Failure in Korea: Present and Future. Korean Circ J. 2016;46:658-664.

9. Moller S, Bernardi M. Interactions of the heart and the liver. Eur Heart J. 2013;34:2804-11.

10. Xanthopoulos A, Starling RC, Kitai T, Triposkiadis F. Heart Failure and Liver Disease: Cardiohepatic Interactions. JACC Heart Fail. 2019;7:87-97.

11. Anstee QM, Mantovani A, Tilg H, Targher G. Risk of cardiomyopathy and cardiac arrhythmias in patients with nonalcoholic fatty liver disease. Nat Rev Gastroenterol Hepatol. 2018;15:425-439.

12. Zhang Z, Wang P, Guo F, Liu X, Luo T, Guan Y, et al. Chronic heart failure in patients with nonalcoholic fatty liver disease: prevalence, clinical features, and relevance. J Int Med Res. 2018;46:3959-3969.

13. Lee J, Lee JS, Park SH, Shin SA, Kim K. Cohort Profile: The National Health Insurance Service-National Sample Cohort (NHIS-NSC), South Korea. Int J Epidemiol. 2017;46:e15.

14. Consultation WHOE. Appropriate body-mass index for Asian populations and its implications for policy and intervention strategies. Lancet. 2004;363:157-63.

15. Bedogni G, Bellentani S, Miglioli L, Masutti F, Passalacqua M, Castiglione A, et al. The Fatty Liver Index: a simple and accurate predictor of hepatic steatosis in the general population. BMC Gastroenterol. 2006;6:33.

16. Grundy SM, Cleeman JI, Daniels SR, Donato KA, Eckel RH, Franklin BA, et al. Diagnosis and management of the metabolic syndrome: an American Heart Association/National Heart, Lung, and Blood Institute Scientific Statement. Circulation. 2005;112:2735-52.

17. Yang BL, Wu WC, Fang KC, Wang YC, Huo TI, Huang YH, et al. External validation of fatty liver index for identifying ultrasonographic fatty liver in a large-scale cross-sectional study in Taiwan. PLoS One. 2015;10:e0120443.

18. Dhingra R, Gona P, Wang TJ, Fox CS, D'Agostino RB, Sr., Vasan RS. Serum gamma-glutamyl transferase and risk of heart failure in the community. Arterioscler Thromb Vasc Biol. 2010;30:1855-60.

19. Wannamethee SG, Whincup PH, Shaper AG, Lennon L, Sattar N. Gamma-glutamyltransferase, hepatic enzymes, and risk of incident heart failure in older men. Arterioscler Thromb Vasc Biol. 2012;32:830-5.

20. Wang Y, Tuomilehto J, Jousilahti P, Salomaa V, Li B, Antikainen R, et al. Serum gamma-glutamyltransferase and the risk of heart failure in men and women in Finland. Heart. 2013;99:163-7.

21. Jung JY, Park SK, Ryoo JH, Oh CM, Kang JG, Lee JH, et al. Effect of non-alcoholic fatty liver disease on left ventricular diastolic function and geometry in the Korean general population. Hepatol Res. 2017;47:522-532.

22. Trovato FM, Martines GF, Catalano D, Musumeci G, Pirri C, Trovato GM. Echocardiography and NAFLD (non-alcoholic fatty liver disease). Int J Cardiol. 2016;221:275-9.

23. Saad MF, Rewers M, Selby J, Howard G, Jinagouda S, Fahmi S, et al. Insulin resistance and hypertension: the Insulin Resistance Atherosclerosis study. Hypertension. 2004;43:1324-31.

24. Bataller R, Sancho-Bru P, Gines P, Lora JM, Al-Garawi A, Sole M, et al. Activated human hepatic stellate cells express the renin-angiotensin system and synthesize angiotensin II. Gastroenterology. 2003;125:117-25.

25. Reaven GM, Hoffman BB. A role for insulin in the aetiology and course of hypertension? Lancet. 1987;2:435-7.

26. Soleimani M. Insulin resistance and hypertension: new insights. Kidney Int. 2015;87:497-9.

27. Sigala B, McKee C, Soeda J, Pazienza V, Morgan M, Lin Cl, et al. Sympathetic nervous system catecholamines and neuropeptide $Y$ neurotransmitters are upregulated in human NAFLD and modulate the fibrogenic function of hepatic stellate cells. PLoS One. 2013;8:e72928.

28. Warner FJ, Lubel JS, McCaughan GW, Angus PW. Liver fibrosis: a balance of ACEs? Clin Sci (Lond). 2007;113:109-18.

29. Brandes RP. Endothelial dysfunction and hypertension. Hypertension. 2014;64:924-8.

30. Browning JD and Horton JD. Molecular mediators of hepatic steatosis and liver injury. J Clin Invest. 2004;114:147-52.

31. Takahashi T, Watanabe T, Shishido T, Watanabe K, Sugai T, Toshima T, et al. The impact of non-alcoholic fatty liver disease fibrosis score on cardiac prognosis in patients with chronic heart failure. Heart Vessels. 2018;33:733-739.

32. Valbusa F, Agnoletti D, Scala L, Grillo C, Arduini P, Bonapace S, et al. Non-alcoholic fatty liver disease and increased risk of all-cause mortality in elderly patients admitted for acute heart failure. Int J Cardiol. 2018;265:162-168. 
33. Targher G, Byrne CD, Lonardo A, Zoppini G, Barbui C. Non-alcoholic fatty liver disease and risk of incident cardiovascular disease: A metaanalysis. J Hepatol. 2016;65:589-600.

34. VanWagner LB, Wilcox JE, Colangelo LA, Lloyd-Jones DM, Carr JJ, Lima JA, et al. Association of nonalcoholic fatty liver disease with subclinical myocardial remodeling and dysfunction: A population-based study. Hepatology. 2015;62:773-83.

35. Mantovani A, Pernigo M, Bergamini C, Bonapace S, Lipari P, Valbusa F, et al. Heart valve calcification in patients with type 2 diabetes and nonalcoholic fatty liver disease. Metabolism. 2015;64:879-87.

36. Valentova M, von Haehling S, Doehner W, Murin J, Anker SD, Sandek A. Liver dysfunction and its nutritional implications in heart failure. Nutrition. 2013;29:370-8.

37. Sawyer DB. Oxidative stress in heart failure: what are we missing? Am J Med Sci. 2011;342:120-4.

38. Niebauer J, Volk HD, Kemp M, Dominguez M, Schumann RR, Rauchhaus M, et al. Endotoxin and immune activation in chronic heart failure: a prospective cohort study. Lancet. 1999;353:1838-42.

\section{Tables}

Table 1. Baseline characteristics and laboratory findings. 


\begin{tabular}{|c|c|c|c|c|c|c|}
\hline & \multicolumn{4}{|c|}{ FLI quartile } & \multirow[t]{2}{*}{ P-value } & \multirow[t]{2}{*}{ P for trend } \\
\hline & Q1 & Q2 & Q3 & Q4 & & \\
\hline \multicolumn{7}{|l|}{ Baseline characteristics } \\
\hline Age, years & $36.7 \pm 11.4$ & $41.9 \pm 12.4$ & $44.0 \pm 12.4$ & $43.1 \pm 11.3$ & $<0.001$ & $<0.001$ \\
\hline Male sex (\%) & $12,102(15.7)$ & $30,737(39.8)$ & $46,692(60.5)$ & $62,507(81.0)$ & $<0.001$ & $<0.001$ \\
\hline Height, $\mathrm{cm}$ & $161.4 \pm 7.2$ & $163.4 \pm 8.9$ & $165.6 \pm 9.3$ & $168.8 \pm 8.4$ & $<0.001$ & $<0.001$ \\
\hline Weight, Kg & $52.2 \pm 6.1$ & $59.1 \pm 7.3$ & $65.4 \pm 8.3$ & $75.0 \pm 10.4$ & $<0.001$ & $<0.001$ \\
\hline BMI, $\mathrm{Kg} / \mathrm{m}^{2}$ & $20.0 \pm 1.7$ & $22.1 \pm 1.8$ & $23.8 \pm 2.1$ & $26.3 \pm 2.8$ & $<0.001$ & $<0.001$ \\
\hline Waist circumference, $\mathrm{cm}$ & $67.9 \pm 4.9$ & $74.9 \pm 4.8$ & $80.5 \pm 5.1$ & $87.6 \pm 6.5$ & $<0.001$ & $<0.001$ \\
\hline WHR, \% & $0.3 \pm 0.0$ & $0.4 \pm 0.0$ & $0.4 \pm 0.0$ & $0.4 \pm 0.1$ & $<0.001$ & $<0.001$ \\
\hline $\mathrm{SBP}, \mathrm{mmHg}$ & $111.0 \pm 11.1$ & $115.4 \pm 11.1$ & $118.6 \pm 10.6$ & $121.8 \pm 10.0$ & $<0.001$ & $<0.001$ \\
\hline $\mathrm{DBP}, \mathrm{mmHg}$ & $69.4 \pm 8.0$ & $72.0 \pm 7.9$ & $74.0 \pm 7.6$ & $76.3 \pm 7.1$ & $<0.001$ & $<0.001$ \\
\hline Smoking & & & & & $<0.001$ & $<0.001$ \\
\hline Non-smoker & $65,184(84.5)$ & $53,779(69.7)$ & $42,729(55.4)$ & $28,355(36.8)$ & & \\
\hline Ex-smoker & $3,609(4.7)$ & $6,720(8.7)$ & $10,711(13.9)$ & $14,058(18.2)$ & & \\
\hline Current-smoker & $8,350(10.8)$ & $16,638(21.6)$ & $23,713(30.7)$ & $34,732(45.0)$ & & \\
\hline \multicolumn{7}{|l|}{ Alcohol consumption } \\
\hline Amount, g/week & $31.6 \pm 77.4$ & $49.5 \pm 102.2$ & $73.9 \pm 129.6$ & $122.5 \pm 174.0$ & $<0.001$ & $<0.001$ \\
\hline Activity, MET-min/week & $346.7 \pm 350.8$ & $379.7 \pm 382.7$ & $388.8 \pm 388.1$ & $374.6 \pm 373.4$ & $<0.001$ & $<0.001$ \\
\hline \multicolumn{7}{|l|}{ Laboratory findings } \\
\hline AST, IU/L & $19.8 \pm 8.0$ & $21.7 \pm 12.4$ & $23.7 \pm 16.7$ & $29.4 \pm 33.0$ & $<0.001$ & $<0.001$ \\
\hline $\mathrm{ALT}, \mathrm{IU} / \mathrm{L}$ & $14.5 \pm 8.9$ & $17.9 \pm 12.2$ & $22.9 \pm 22.2$ & $36.3 \pm 50.5$ & $<0.001$ & $<0.001$ \\
\hline GGT, IU/L & $14.2 \pm 5.6$ & $19.1 \pm 9.9$ & $28.1 \pm 20.3$ & $60.3 \pm 62.4$ & $<0.001$ & $<0.001$ \\
\hline Hemoglobin, g/dL & $13.0 \pm 1.4$ & $13.6 \pm 1.6$ & $14.2 \pm 1.6$ & $14.9 \pm 1.4$ & $<0.001$ & $<0.001$ \\
\hline Fasting glucose, mg/dL & $87.9 \pm 9.5$ & $90.3 \pm 10.2$ & $92.2 \pm 10.7$ & $94.8 \pm 11.4$ & $<0.001$ & $<0.001$ \\
\hline Total cholesterol, mg/dL & $176.9 \pm 33.6$ & $187.1 \pm 35.9$ & $196.2 \pm 39.3$ & $207.0 \pm 40.7$ & $<0.001$ & $<0.001$ \\
\hline Triglyceride, mg/dL & $62.6 \pm 22.5$ & $88.0 \pm 34.0$ & $119.9 \pm 53.6$ & $197.6 \pm 132.6$ & $<0.001$ & $<0.001$ \\
\hline HDL cholesterol, mg/dL & $64.1 \pm 24.8$ & $59.3 \pm 19.9$ & $55.5 \pm 25.8$ & $51.7 \pm 32.0$ & $<0.001$ & $<0.001$ \\
\hline LDL cholesterol, mg/dL & $109.4 \pm 257.2$ & $113.6 \pm 143.5$ & $121.2 \pm 134.2$ & $122.6 \pm 129.0$ & $<0.001$ & $<0.001$ \\
\hline Creatinine, mg/dL & $0.9 \pm 1.0$ & $1.0 \pm 1.1$ & $1.0 \pm 1.1$ & $1.1 \pm 1.2$ & $<0.001$ & $<0.001$ \\
\hline $\mathrm{GFR}, \mathrm{mL} / \mathrm{min} / 1.73 \mathrm{~m}^{2}$ & $90.2 \pm 27.9$ & $90.3 \pm 28.0$ & $92.6 \pm 29.4$ & $99.7 \pm 30.2$ & $<0.001$ & $<0.001$ \\
\hline
\end{tabular}

ALT: alanine aminotransferase, AST: aspartate aminotransferase, BMI: body mass index, DBP: diastolic blood pressure, GFR: glomerular filtration rate, GGT: gamma-glutamyl transferase, HDL: high-density lipoprotein, LDL: low-density lipoprotein, SBP: systolic blood pressure, WHR: waist-hip ratio

Table 2. The Univariate analysis of new-onset heart failure. 


\begin{tabular}{|c|c|c|c|c|c|c|c|}
\hline \multirow[t]{2}{*}{ Characteristics } & \multirow[t]{2}{*}{ Value } & \multirow[t]{2}{*}{$\mathrm{N}$} & \multirow[t]{2}{*}{$\mathrm{HF}(\%)$} & \multirow[t]{2}{*}{$\mathrm{HR}$} & \multicolumn{2}{|c|}{$95 \%$ CI } & \multirow[t]{2}{*}{ P-value } \\
\hline & & & & & Lower & Upper & \\
\hline Total & Total & 308,578 & $2,532(0.8)$ & & & & \\
\hline \multirow[t]{2}{*}{ Sex } & Female & 156,540 & $1,263(0.8)$ & \multicolumn{4}{|c|}{ Reference } \\
\hline & Male & 152,038 & $1,269(0.8)$ & 1.113 & 1.030 & 1.204 & 0.007 \\
\hline \multirow[t]{6}{*}{ Age, year } & $20-34$ & 99,774 & $119(0.1)$ & \multicolumn{4}{|c|}{ Reference } \\
\hline & $35-49$ & 130,099 & $637(0.5)$ & 3.908 & 3.213 & 4.753 & $<0.001$ \\
\hline & $50-64$ & 65,907 & $1,067(1.6)$ & 12.536 & 10.371 & 15.153 & $<0.001$ \\
\hline & $65-74$ & 10,374 & $497(4.8)$ & 35.791 & 29.298 & 43.722 & $<0.001$ \\
\hline & $\geq 75$ & 2,424 & $212(8.7)$ & 75.652 & 60.429 & 94.711 & $<0.001$ \\
\hline & Continuous & & & 1.087 & 1.084 & 1.090 & $<0.001$ \\
\hline \multirow[t]{6}{*}{ BMI, $\mathrm{Kg} / \mathrm{m}^{2}$} & $<18.5$ & 16,662 & $101(0.6)$ & 1.075 & 0.870 & 1.328 & 0.505 \\
\hline & $18.5-19.9$ & 33,181 & $177(0.5)$ & 0.929 & 0.786 & 1.098 & 0.390 \\
\hline & $20-22.4$ & 89,515 & $625(0.7)$ & \multicolumn{4}{|c|}{ Reference } \\
\hline & $22.5-24.9$ & 90,961 & $814(0.9)$ & 1.115 & 1.005 & 1.238 & 0.041 \\
\hline & $\geq 25$ & 78,259 & $815(1.0)$ & 1.417 & 1.276 & 1.573 & $<0.001$ \\
\hline & Continuous & & & 1.058 & 1.045 & 1.072 & $<0.001$ \\
\hline \multirow[t]{3}{*}{ Smoking } & Non-smoker & 190,047 & $1,599(0.8)$ & \multicolumn{4}{|c|}{ Reference } \\
\hline & Ex-smoker & 35,098 & $320(0.9)$ & 0.934 & 0.812 & 1.074 & 0.340 \\
\hline & Current-smoker & 83,433 & $613(0.7)$ & 1.120 & 0.997 & 1.259 & 0.057 \\
\hline \multirow[t]{4}{*}{ Alcohol consumption, g/week } & 0 & 149,779 & $1,557(1.0)$ & \multicolumn{4}{|c|}{ Reference } \\
\hline & $<=140$ & 110,537 & $626(0.6)$ & 0.882 & 0.798 & 0.975 & 0.014 \\
\hline & $>140$ & 48,262 & $349(0.7)$ & 0.991 & 0.871 & 1.126 & 0.890 \\
\hline & Continuous & & & 1.000 & 1.000 & 1.000 & 0.960 \\
\hline \multirow[t]{3}{*}{$\mathrm{SBP}, \mathrm{mmHg}$} & $<120$ & 165,193 & $1,019(0.6)$ & \multicolumn{4}{|c|}{ Reference } \\
\hline & $120-139$ & 143,385 & $1,513(1.1)$ & 1.295 & 1.195 & 1.404 & $<0.001$ \\
\hline & Continuous & & & 1.013 & 1.010 & 1.017 & $<0.001$ \\
\hline \multirow[t]{3}{*}{$\mathrm{DBP}, \mathrm{mmHg}$} & $<80$ & 210,638 & $1,513(0.7)$ & \multicolumn{4}{|c|}{ Reference } \\
\hline & $80-89$ & 97,940 & $1,019(1.0)$ & 1.220 & 1.126 & 1.322 & $<0.001$ \\
\hline & Continuous & & & 1.016 & 1.011 & 1.022 & $<0.001$ \\
\hline \multirow[t]{3}{*}{ FBS, mg/dL } & $<100$ & 246,415 & $1,876(0.8)$ & \multicolumn{4}{|c|}{ Reference } \\
\hline & $100-125$ & 62,163 & $656(1.1)$ & 1.042 & 0.953 & 1.140 & 0.370 \\
\hline & Continuous & & & 1.003 & 1.000 & 1.007 & 0.058 \\
\hline \multirow[t]{4}{*}{ Activity, MET-min/week } & $<500$ & 221107 & $1871(0.8)$ & & & & \\
\hline & $500-999$ & 65361 & $453(0.7)$ & 0.877 & 0.791 & 0.972 & 0.013 \\
\hline & $\geq 1000$ & 22110 & $208(0.9)$ & 0.890 & 0.770 & 1.027 & 0.110 \\
\hline & Continuous & & & 1.000 & 0.999 & 1.000 & 0.210 \\
\hline Waist circumference, $\mathrm{cm}$ & Continuous & & & 1.027 & 1.022 & 1.032 & $<0.001$ \\
\hline Total cholesterol, mg/dL & Continuous & & & 1.001 & 1.000 & 1.002 & $<0.001$ \\
\hline Triglyceride, mg/dL & Continuous & & & 1.001 & 1.000 & 1.001 & $<0.001$ \\
\hline HDL-cholesterol, mg/dL & Continuous & & & 0.997 & 0.995 & 0.999 & 0.014 \\
\hline LDL-cholesterol, mg/dL & Continuous & & & 1.000 & 1.000 & 1.000 & 0.290 \\
\hline
\end{tabular}

BMI: body mass index, CI: confidential interval, DBP: diastolic blood pressure, FBS: fasting blood glucose, HDL: highdensity lipoprotein, HF: heart failure, HR: hazard ration, LDL: low-density lipoprotein, MET: the metabolic equivalent of task, SBP: systolic blood pressure 
Table 3. Association between the fatty liver index and new-onset heart failure.

\begin{tabular}{|c|c|c|c|c|c|c|c|c|c|c|c|}
\hline \multirow[t]{2}{*}{ FLI } & \multirow[t]{2}{*}{ N. } & \multirow{2}{*}{$\begin{array}{c}\text { New-onset } \\
\text { HF }\end{array}$} & \multicolumn{3}{|c|}{ Univariate } & \multicolumn{3}{|c|}{ Model 1* } & \multicolumn{3}{|c|}{ Model $2^{\dagger}$} \\
\hline & & & HR & $95 \%$ CI & $\begin{array}{c}\text { P- } \\
\text { value }\end{array}$ & $\mathrm{HR}$ & $95 \%$ CI & $\begin{array}{c}\text { P- } \\
\text { value }\end{array}$ & HR & $95 \%$ CI & $\begin{array}{c}\text { P- } \\
\text { value }\end{array}$ \\
\hline $\begin{array}{l}\text { FLI quartile } \\
\text { Q1 (0 - 4.9) }\end{array}$ & 77,143 & $307(0.4)$ & & Reference & & & Referenc & & & Reference & \\
\hline $\begin{array}{l}\text { Q2 (5.0 - } \\
12.5)\end{array}$ & 77,137 & $543(0.7)$ & 1.691 & $\begin{array}{l}1.470- \\
1.945\end{array}$ & $<0.001$ & 1.104 & $\begin{array}{l}0.958- \\
1.271\end{array}$ & 0.171 & 1.071 & $\begin{array}{l}0.930- \\
1.234\end{array}$ & 0.339 \\
\hline $\begin{array}{l}\text { Q3 (12.6 - } \\
31.0)\end{array}$ & 77,153 & $792(1.0)$ & 2.409 & $\begin{array}{l}2.112- \\
2.748\end{array}$ & $<0.001$ & 1.386 & $\begin{array}{l}1.212- \\
1.586\end{array}$ & $<0.001$ & 1.314 & $\begin{array}{l}1.147- \\
1.504\end{array}$ & $<0.001$ \\
\hline $\mathrm{Q} 4(>31.0)$ & 77,145 & $890(1.2)$ & 2.709 & $\begin{array}{l}2.380- \\
3.085\end{array}$ & $<0.001$ & 1.857 & $\begin{array}{l}1.620- \\
2.127\end{array}$ & $<0.001$ & 1.710 & $\begin{array}{l}1.489- \\
1.964\end{array}$ & $<0.001$ \\
\hline
\end{tabular}

${ }^{*}$ Cox proportional hazard models including age, and sex as covariates

${ }^{\dagger}$ Cox proportional hazard models including age, sex, smoking, amount of alcohol drinking, activity, systolic blood pressure, diastolic blood pressure, fasting blood glucose, cholesterol, and fatty liver index as covariates.

CI: confidential interval, FLI: fatty liver index, HR: hazard ratio

\section{Supplementary Table}

Supplementary Table 1. Association between the fatty liver index and new-onset heart failure.

\begin{tabular}{|c|c|c|c|c|c|c|c|c|c|c|c|}
\hline \multirow[t]{3}{*}{ FLI } & \multirow[t]{3}{*}{ N. } & \multirow{3}{*}{$\begin{array}{c}\text { New- } \\
\text { onset HF }\end{array}$} & \multicolumn{3}{|c|}{ Univariate } & \multicolumn{3}{|c|}{ Model 1* } & \multicolumn{3}{|c|}{ Model $2^{\dagger}$} \\
\hline & & & HR & $95 \%$ & $\mathrm{P}-$ & $\mathrm{HR}$ & $95 \%$ & $\mathrm{P}-$ & $\mathrm{HR}$ & $95 \%$ & $\mathrm{P}-$ \\
\hline & & & & CI & value & & CI & value & & CI & value \\
\hline \multicolumn{12}{|l|}{$\begin{array}{l}\text { FLI criteria } \\
1\end{array}$} \\
\hline $\begin{array}{l}0 \leq \text { FLI }< \\
30\end{array}$ & 228,136 & $1,608(0.7)$ & Reference & & & Reference & & & Reference & & \\
\hline $\begin{array}{l}30 \leq \mathrm{FLI}< \\
60\end{array}$ & 54,563 & $614(1.1)$ & 1.538 & $\begin{array}{l}1.402- \\
1.688\end{array}$ & $<0.001$ & 1.376 & $\begin{array}{l}1.250- \\
1.514\end{array}$ & $<0.001$ & 1.320 & $\begin{array}{l}1.198- \\
1.453\end{array}$ & $<0.001$ \\
\hline FLI $\geq 60$ & 25,879 & $310(1.2)$ & 1.669 & $\begin{array}{l}1.478- \\
1.885\end{array}$ & $<0.001$ & 1.961 & $\begin{array}{l}1.727- \\
2.227\end{array}$ & $<0.001$ & 1.849 & $\begin{array}{l}1.622- \\
2.106\end{array}$ & $<0.001$ \\
\hline \multicolumn{12}{|l|}{$\begin{array}{l}\text { FLI criteria } \\
2 \ddagger\end{array}$} \\
\hline $\begin{array}{l}\text { Low } \\
\text { probability }\end{array}$ & 178,464 & $10,20(0.6)$ & Reference & & & Reference & & & Reference & & \\
\hline $\begin{array}{l}\text { Intermediate } \\
\text { probability }\end{array}$ & 46,892 & $480(1.0)$ & 1.735 & $\begin{array}{l}1.556- \\
1.933\end{array}$ & $<0.001$ & 1.233 & $\begin{array}{l}1.105- \\
1.376\end{array}$ & $<0.001$ & 1.196 & $\begin{array}{l}1.072- \\
1.334\end{array}$ & 0.001 \\
\hline $\begin{array}{l}\text { High } \\
\text { probability }\end{array}$ & 83,222 & $1,032(1.2)$ & 2.104 & $\begin{array}{l}1.930- \\
2.294\end{array}$ & $<0.001$ & 1.631 & $\begin{array}{c}1.496- \\
1.779\end{array}$ & $<0.001$ & 1.557 & $\begin{array}{l}1.423- \\
1.704\end{array}$ & $<0.001$ \\
\hline
\end{tabular}

${ }^{*}$ Cox proportional hazard models including age, and sex as covariates

${ }^{\dagger}$ Cox proportional hazard models including age, sex, smoking, amount of alcohol consumption, physical activity, systolic blood pressure, diastolic blood pressure, fasting blood glucose, cholesterol, and the fatty liver index as covariates.

${ }^{\ddagger}$ Low probability: $0 \leq$ FLI $<25$ for male, $0 \leq$ FLI $<10$ for female; intermediate probability: $25 \leq$ FLI $<35$ for male, $10 \leq$ FLI $<20$ for female; high probability: FLI $\geq 35$ for male, FLI $\geq 20$ for female

CI: confidential interval, FLI: fatty liver index, HR: hazard ratio

\section{Figures}


556,884 participants who underwent the National Health checkups from Jan. 2009 to Dec. 2014

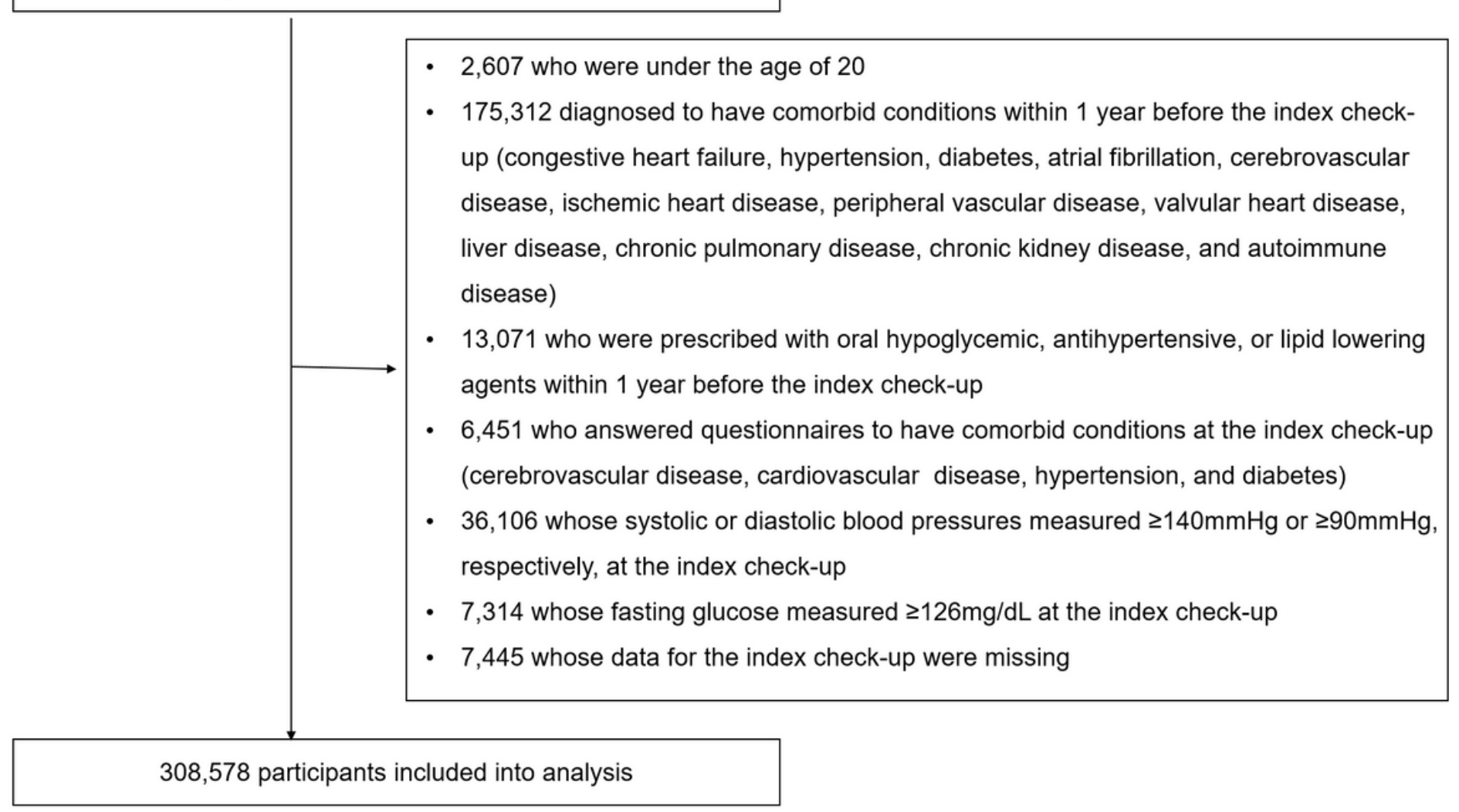

Figure 1

The overview of the study population. 


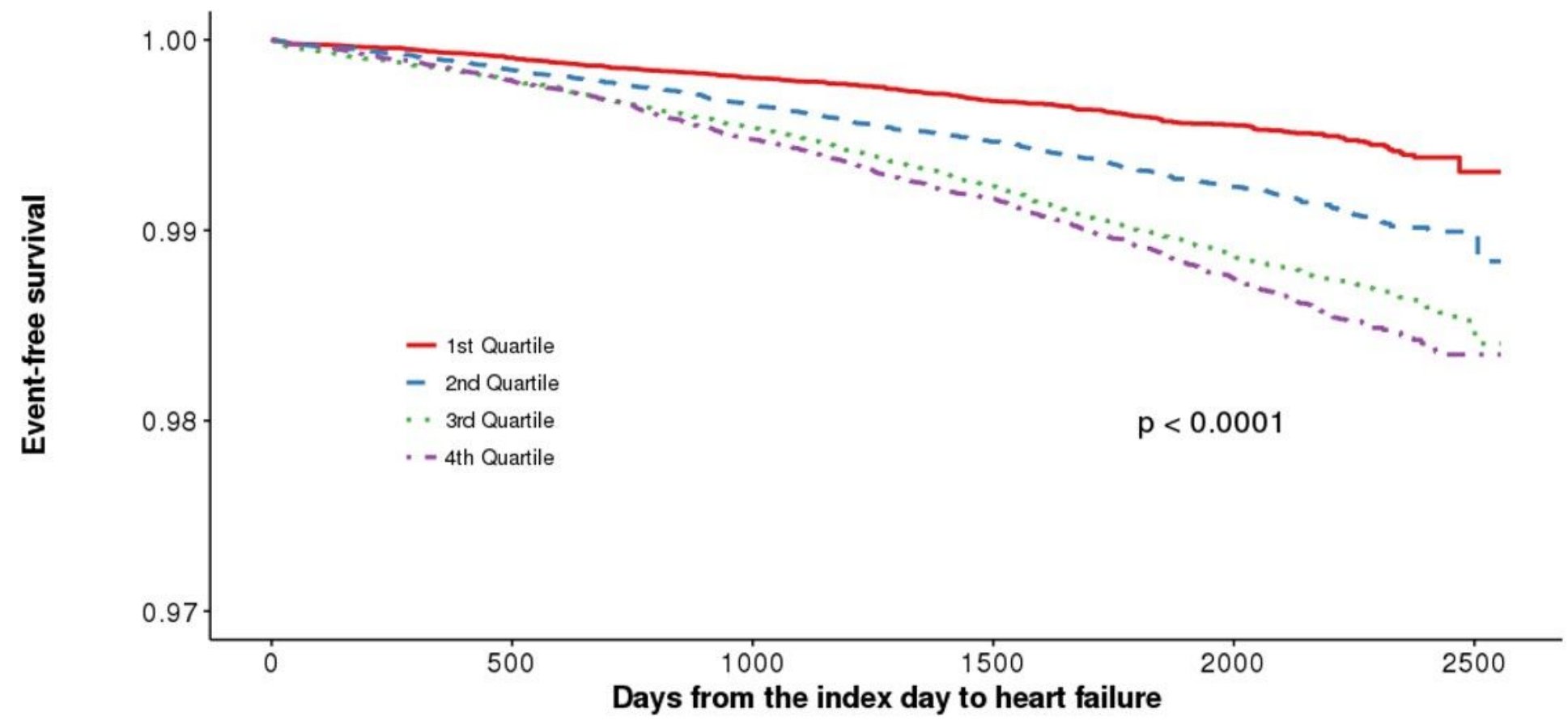

Number at risk

\begin{tabular}{|c|c|c|c|c|c|}
\hline $\begin{array}{r}\text { 1st Quartile- } 77143 \\
\text { 2nd Quartile- } 77137 \\
\text { 3rd Quartile- } 77153 \\
\text { 4th Quartile- } 77145\end{array}$ & $\begin{array}{l}73291 \\
74041 \\
74404 \\
74148\end{array}$ & $\begin{array}{l}64459 \\
66734 \\
67701 \\
67523\end{array}$ & $\begin{array}{l}52624 \\
55826 \\
57300 \\
57230\end{array}$ & $\begin{array}{l}33140 \\
36734 \\
38947 \\
38927\end{array}$ & $\begin{array}{l}331 \\
636 \\
820 \\
701\end{array}$ \\
\hline
\end{tabular}

Figure 2

Cumulative incidence of new-onset heart failure according to the quartiles. Statistical significance using the log-rank test. 


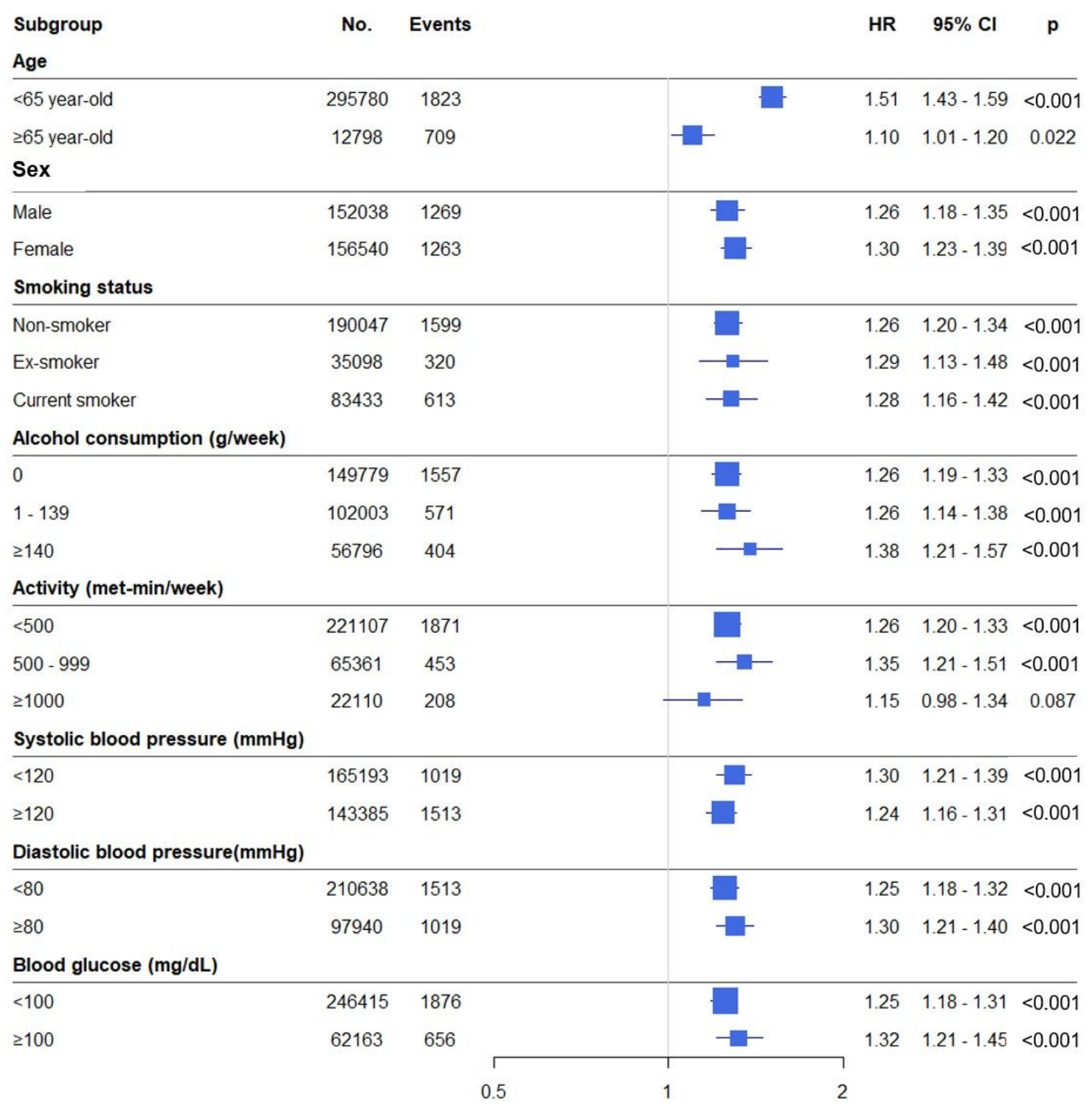

\section{Figure 3}

The Forest plots of hazard ratios for new-onset heart failure stratified by various clinical characteristics. $\mathrm{Cl}=$ confidence interval; $\mathrm{HR}=$ hazard ratio. 